Bundesgesundheitsbl 2014 · 57:594-594

DOI 10.1007/s00103-014-1948-x

๑) Springer-Verlag Berlin Heidelberg 2014

Mitteilungen des Arbeitskreises Blut des Bundesministeriums für Gesundheit

\title{
Zulassung von Spendewilligen mit körperlichen und/oder geistigen Einschränkungen
}

\section{Stellungnahme}

\section{Bei der 77. Sitzung des Arbeitskreises Blut am 24./25.02.2014 wurde folgende Stellungnahme (S 13) verabschiedet:}

Jeder Blutspender erbringt einen wichtigen Beitrag zur Sicherstellung der Blutversorgung in Deutschland. Information, Aufklärung und Voruntersuchung von Spendewilligen haben eine hohe Bedeutung und Priorität.

Im Sinne der rechtlichen und ethischen Gleichstellung von Menschen mit körperlichen und geistigen Einschränkungen muss eine Beteiligung dieser Personen an der Blutspende grundsätzlich ermöglicht und gefördert werden. Entsprechend ist die Eignung zur Blutspende bei Spendewilligen mit körperlichen und geistigen Einschränkungen in gleicher Weise zu überprüfen wie bei Spendewilligen ohne solche Einschränkungen.

Die Richtlinien zur Gewinnung von Blut und Blutbestandteilen und zur Anwendung von Blutprodukten (Hämotherapie) legen die Kriterien zur Spenderauswahl und somit zur Gewinnung von Blut- und Blutbestandteilen fest. Gemäß diesen Richtlinien sind die beiden nachfolgenden Prinzipien immer gleichzeitig und gleichermaßen zu prüfen und umzusetzen:

- der Erhalt des Wohls der Spendewilligen und

- die Unbedenklichkeit und optimale Sicherheit des gesammelten Blutes.
Gemäß den geltenden Richtlinien zur Gewinnung von Blut und Blutbestandteilen und zur Anwendung von Blutprodukten (Hämotherapie) der Bundesärztekammer ist bei jeder Voruntersuchung und Zulassung eines potenziellen Spenders zu einer Blutspende zu prüfen,

1. ob mit dieser Spende für den Betroffenen ein zusätzliches erhöhtes Risiko einhergeht und

2. ob die Qualität und Sicherheit des entstehenden Arzneimittels beeinträchtigt ist.

Diese Umsetzung der Richtlinien kann insofern nur medizinischen und arzneimittelrechtlichen Kriterien folgen und muss bei Spendewilligen ohne und mit körperlichen und/oder geistigen Einschränkungen immer zu einer abgewogenen individuellen ärztlichen Entscheidung führen. Dabei ist vor jeder Blutspende festzustellen, ob eine erhöhte Gefährdung des Spenders anzunehmen ist oder ob die Einsichts- und Einwilligungsfähigkeit eingeschränkt ist. Zudem muss ausgeschlossen werden, dass eine Einschränkung der Qualität und Sicherheit des Arzneimittels zu erwarten ist (wie z. B. durch Risikoexpositionen). In solchen Fällen kann eine Zulassung zur Spende nicht erfolgen.

Das Hinzuziehen einer Vertrauensperson zum gemäß $\$ 6$ Transfusionsgesetz verbindlichen vertraulichen Selbstausschluss ist nicht zulässig.
Nach den Vorgaben der Hämotherapie-Richtlinien muss die ärztliche Person beurteilen, ob die spendewillige Person in der Lage ist, die Inhalte der Befragung und der Einverständniserklärung angemessen zu verstehen. Ebenso darf bei Spendewilligen mit körperlichen Einschränkungen ein erhöhtes bzw. zusätzliches Risiko für Spendekomplikationen nicht in Kauf genommen werden.

Grundsätzlich kann eine ganze Gruppe von Menschen mit Einschränkungen, wie z. B. Erblindete oder Gehörlose, nicht insgesamt zur Blutspende zugelassen oder kollektiv von der Blutspende ausgeschlossen werden. Für jeden Spendewilligen erfordert die Zulassung zur Blutspende stets die ärztliche Einzelfallentscheidung als eine aktuelle Beurteilung unter Beachtung der Hämotherapie-Richtlinien.

Für den Arbeitskreis Blut

Dr. R. Offergeld, Vorsitzende

Prof. Dr. R. Burger 